\title{
Positive solutions to a system of semipositone fractional boundary value problems
}

\author{
Rodica Luca ${ }^{1 *}$ and Alexandru Tudorache ${ }^{2}$
}

\section{"Correspondence:}

rlucatudor@yahoo.com

'Department of Mathematics, Gh

Asachi Technical University, lasi,

700506, Romania

Full list of author information is

available at the end of the article

\begin{abstract}
We study the existence of positive solutions for a system of nonlinear Riemann-Liouville fractional differential equations with sign-changing nonlinearities, subject to integral boundary conditions.
\end{abstract}

MSC: $34 \mathrm{~A} 08 ; 45 \mathrm{G} 15$

Keywords: Riemann-Liouville fractional differential equation; integral boundary conditions; positive solutions

\section{Introduction}

We consider the system of nonlinear ordinary fractional differential equations

$$
\begin{cases}D_{0+}^{\alpha} u(t)+\lambda f(t, u(t), v(t))=0, & t \in(0,1), n-1<\alpha \leq n, \\ D_{0+}^{\beta} v(t)+\mu g(t, u(t), v(t))=0, & t \in(0,1), m-1<\beta \leq m,\end{cases}
$$

with the integral boundary conditions

$$
\begin{cases}u(0)=u^{\prime}(0)=\cdots=u^{(n-2)}(0)=0, & u(1)=\int_{0}^{1} u(s) d H(s), \\ v(0)=v^{\prime}(0)=\cdots=v^{(m-2)}(0)=0, & v(1)=\int_{0}^{1} v(s) d K(s),\end{cases}
$$

where $n, m \in \mathbb{N}, n, m \geq 3, D_{0_{+}}^{\alpha}$, and $D_{0+}^{\beta}$ denote the Riemann-Liouville derivatives of orders $\alpha$ and $\beta$, respectively, the integrals from (BC) are Riemann-Stieltjes integrals and $f, g$ are sign-changing continuous functions (that is, we have a so-called system of semipositone boundary value problems). These boundary conditions include multi-point and integral boundary conditions and the sum of these in a single framework.

Fractional differential equations describe many phenomena in various fields of engineering and scientific disciplines such as physics, biophysics, chemistry, biology, economics, control theory, signal and image processing, aerodynamics, viscoelasticity, electromagnetics, and so on (see [1-7]). Integral boundary conditions arise in thermal conduction problems, semiconductor problems, and hydrodynamic problems.

By using a nonlinear alternative of Leray-Schauder type, we present intervals for parameters $\lambda$ and $\mu$ such that the above problem (S)-(BC) has at least one positive solution. By a positive solution of problem (S)-(BC) we mean a pair of functions $(u, v) \in$ $C([0,1]) \times C([0,1])$ satisfying (S) and $(\mathrm{BC})$ with $u(t) \geq 0, v(t) \geq 0$ for all $t \in[0,1]$ and $u(t)>0, v(t)>0$ for all $t \in(0,1)$. In the case when $f$ and $g$ are nonnegative, the above 
problem (S)-(BC) has been investigated in [8] by using the Guo-Krasnosel'skii fixed point theorem. The system (S) with $\lambda=\mu=1$, and with $f(t, u, v)$ and $g(t, u, v)$ replaced by $\widetilde{f}(t, v)$ and $\widetilde{g}(t, u)$, respectively, with the boundary conditions (BC), was studied in [9]. In [9], the authors obtained the existence and multiplicity of positive solutions $(u(t) \geq 0, v(t) \geq 0$ for all $\left.t \in[0,1], \sup _{t \in[0,1]} u(t)>0, \sup _{t \in[0,1]} v(t)>0\right)$ by applying some theorems from the fixed point index theory. We would also like to mention the paper [10], where the authors investigated the existence and multiplicity of positive solutions of the semipositone system (S) with $\alpha=\beta$ and the boundary conditions $u^{(i)}(0)=v^{(i)}(0)=0, i=0, \ldots, n-2, u(1)=a v(\xi)$, $v(1)=b v(\eta), \xi, \eta \in(0,1)$, and $0<a b \xi \eta<1$.

The paper is organized as follows. Section 2 contains some preliminaries and lemmas. The main theorem is presented in Section 3 and, finally, in Section 4, two examples are given to support the new result.

\section{Auxiliary results}

We present here the definitions, some lemmas from the theory of fractional calculus, and some auxiliary results that will be used to prove our main theorem.

Definition 2.1 The (left-sided) fractional integral of order $\alpha>0$ of a function $f:(0, \infty) \rightarrow$ $\mathbb{R}$ is given by

$$
\left(I_{0+}^{\alpha} f\right)(t)=\frac{1}{\Gamma(\alpha)} \int_{0}^{t}(t-s)^{\alpha-1} f(s) d s, \quad t>0,
$$

provided the right-hand side is pointwise defined on $(0, \infty)$, where $\Gamma(\alpha)$ is the Euler gamma function defined by $\Gamma(\alpha)=\int_{0}^{\infty} t^{\alpha-1} e^{-t} d t, \alpha>0$.

Definition 2.2 The Riemann-Liouville fractional derivative of order $\alpha \geq 0$ for a function $f:(0, \infty) \rightarrow \mathbb{R}$ is given by

$$
\left(D_{0+}^{\alpha} f\right)(t)=\left(\frac{d}{d t}\right)^{n}\left(I_{0+}^{n-\alpha} f\right)(t)=\frac{1}{\Gamma(n-\alpha)}\left(\frac{d}{d t}\right)^{n} \int_{0}^{t} \frac{f(s)}{(t-s)^{\alpha-n+1}} d s, \quad t>0,
$$

where $n=\llbracket \alpha \rrbracket+1$, provided that the right-hand side is pointwise defined on $(0, \infty)$.

The notation $\llbracket \alpha \rrbracket$ stands for the largest integer not greater than $\alpha$. We also denote the Riemann-Liouville fractional derivative of $f$ by $D_{0+}^{\alpha} f(t)$. If $\alpha=m \in \mathbb{N}$ then $D_{0+}^{m} f(t)=f^{(m)}(t)$ for $t>0$, and if $\alpha=0$ then $D_{0+}^{0} f(t)=f(t)$ for $t>0$.

Lemma 2.1 ([4]) Let $\alpha>0$ and $n=\llbracket \alpha \rrbracket+1$ for $\alpha \notin \mathbb{N}$ and $n=\alpha$ for $\alpha \in \mathbb{N}$; that is, $n$ is the smallest integer greater than or equal to $\alpha$. Then the solutions of the fractional differential equation $D_{0+}^{\alpha} u(t)=0,0<t<1$, are

$$
u(t)=c_{1} t^{\alpha-1}+c_{2} t^{\alpha-2}+\cdots+c_{n} t^{\alpha-n}, \quad 0<t<1,
$$

where $c_{1}, c_{2}, \ldots, c_{n}$ are arbitrary real constants.

Lemma 2.2 $([4,8])$ Let $\alpha>0, n$ be the smallest integer greater than or equal to $\alpha(n-1<$ $\alpha \leq n)$ and $y \in L^{1}(0,1)$. The solutions of the fractional equation $D_{0_{+}}^{\alpha} u(t)+y(t)=0,0<t<1$, 
are

$$
u(t)=-\frac{1}{\Gamma(\alpha)} \int_{0}^{t}(t-s)^{\alpha-1} y(s) d s+c_{1} t^{\alpha-1}+\cdots+c_{n} t^{\alpha-n}, \quad 0<t<1
$$

where $c_{1}, c_{2}, \ldots, c_{n}$ are arbitrary real constants.

We consider now the fractional differential equation

$$
D_{0+}^{\alpha} u(t)+z(t)=0, \quad 0<t<1, n-1<\alpha \leq n,
$$

with the integral boundary conditions

$$
u(0)=u^{\prime}(0)=\cdots=u^{(n-2)}(0)=0, \quad u(1)=\int_{0}^{1} u(s) d H(s)
$$

where $n \in \mathbb{N}, n \geq 3$, and $H:[0,1] \rightarrow \mathbb{R}$ is a function of the bounded variation.

By using Lemma 2.2, after some computations, we obtain the following lemma.

Lemma 2.3 ([9]) If $H:[0,1] \rightarrow \mathbb{R}$ is a function of bounded variation, $\Delta_{1}=1-$ $\int_{0}^{1} s^{\alpha-1} d H(s) \neq 0$ and $z \in C([0,1])$, then the solution of problem $(1)-(2)$ is $u(t)=\int_{0}^{1} G_{1}(t, s) \times$ $z(s) d s$, where

$$
\begin{aligned}
& G_{1}(t, s)=g_{1}(t, s)+\frac{t^{\alpha-1}}{\Delta_{1}} \int_{0}^{1} g_{1}(\tau, s) d H(\tau), \quad(t, s) \in[0,1] \times[0,1], \\
& g_{1}(t, s)=\frac{1}{\Gamma(\alpha)} \begin{cases}t^{\alpha-1}(1-s)^{\alpha-1}-(t-s)^{\alpha-1}, & 0 \leq s \leq t \leq 1 \\
t^{\alpha-1}(1-s)^{\alpha-1}, & 0 \leq t \leq s \leq 1\end{cases}
\end{aligned}
$$

Lemma 2.4 The function $g_{1}$ given by (4) has the properties

(a) $g_{1}:[0,1] \times[0,1] \rightarrow \mathbb{R}_{+}$is a continuous function, $g_{1}(t, s) \geq 0$ for all $(t, s) \in[0,1] \times[0,1]$ and $g_{1}(t, s)>0$ for all $(t, s) \in(0,1) \times(0,1)$.

(b) $g_{1}(t, s) \leq h_{1}(s)$ for all $(t, s) \in[0,1] \times[0,1]$, where $h_{1}(s)=\frac{s(1-s)^{\alpha-1}}{\Gamma(\alpha-1)}$.

(c) $g_{1}(t, s) \geq k_{1}(t) h_{1}(s)$ for all $(t, s) \in[0,1] \times[0,1]$, where

$$
k_{1}(t)=\min \left\{\frac{(1-t) t^{\alpha-2}}{\alpha-1}, \frac{t^{\alpha-1}}{\alpha-1}\right\}= \begin{cases}\frac{t^{\alpha-1}}{\alpha-1}, & 0 \leq t \leq \frac{1}{2} \\ \frac{(1-t) t^{\alpha-2}}{\alpha-1}, & \frac{1}{2} \leq t \leq 1\end{cases}
$$

Proof The first part (a) is evident. For the second part (b), see [11].

For part (c), for $s \leq t$, we obtain

$$
\begin{aligned}
g_{1}(t, s) & =\frac{1}{\Gamma(\alpha)}\left[t^{\alpha-1}(1-s)^{\alpha-1}-(t-s)^{\alpha-1}\right]=\frac{1}{\Gamma(\alpha)}\left[(t-t s)^{\alpha-1}-(t-s)^{\alpha-1}\right] \\
& =\frac{1}{\Gamma(\alpha)}\left[(t-t s)^{\alpha-1}-(t-s)^{\alpha-2}(t-s)\right] \\
& \geq \frac{1}{\Gamma(\alpha)}\left[(t-t s)^{\alpha-2}(t-t s)-(t-t s)^{\alpha-2}(t-s)\right] \\
& =\frac{1}{\Gamma(\alpha)} t^{\alpha-2}(1-t) s(1-s)^{\alpha-2} \geq \frac{t^{\alpha-2}(1-t)}{\alpha-1} \cdot \frac{s(1-s)^{\alpha-1}}{\Gamma(\alpha-1)} .
\end{aligned}
$$


If $s \geq t$, we have

$$
g_{1}(t, s)=\frac{1}{\Gamma(\alpha)} t^{\alpha-1}(1-s)^{\alpha-1} \geq \frac{1}{\alpha-1} \cdot \frac{t^{\alpha-1}}{s} \cdot \frac{s(1-s)^{\alpha-1}}{\Gamma(\alpha-1)} \geq \frac{t^{\alpha-1}}{\alpha-1} \cdot \frac{s(1-s)^{\alpha-1}}{\Gamma(\alpha-1)} .
$$

Therefore, we deduce that $g_{1}(t, s) \geq k_{1}(t) h_{1}(s)$, where $k_{1}(t)$ is defined in (5).

Lemma 2.5 ([9]) If $H:[0,1] \rightarrow \mathbb{R}$ is a nondecreasing function and $\Delta_{1}>0$, then the Green's function $G_{1}$ of problem (1)-(2) given by (3) is continuous on $[0,1] \times[0,1]$ and satisfies $G_{1}(t, s) \geq 0$ for all $(t, s) \in[0,1] \times[0,1], G_{1}(t, s)>0$ for all $(t, s) \in(0,1) \times(0,1)$. Moreover, if $z \in C([0,1])$ satisfies $z(t) \geq 0$ for all $t \in[0,1]$, then the unique solution $u$ of problem (1)(2) satisfies $u(t) \geq 0$ for all $t \in[0,1]$.

Lemma 2.6 Assume that $H:[0,1] \rightarrow \mathbb{R}$ is a nondecreasing function and $\Delta_{1}>0$. Then the Green's function $G_{1}$ of problem (1)-(2) satisfies the inequalities:

(a) $G_{1}(t, s) \leq J_{1}(s), \forall(t, s) \in[0,1] \times[0,1]$, where

$$
J_{1}(s)=\tau_{1} h_{1}(s), \quad \tau_{1}=1+\frac{1}{\Delta_{1}} \int_{0}^{1} d H(\tau)=1+\frac{1}{\Delta_{1}}(H(1)-H(0)) .
$$

(b) $G_{1}(t, s) \geq \gamma_{1}(t) J_{1}(s), \forall(t, s) \in[0,1] \times[0,1]$, where

$$
\gamma_{1}(t)=\frac{1}{\tau_{1}}\left(k_{1}(t)+\frac{t^{\alpha-1}}{\Delta_{1}} \int_{0}^{1} k_{1}(\tau) d H(\tau)\right) .
$$

Proof (a) We have

$$
\begin{aligned}
G_{1}(t, s) & =g_{1}(t, s)+\frac{t^{\alpha-1}}{\Delta_{1}} \int_{0}^{1} g_{1}(\tau, s) d H(\tau) \\
& \leq h_{1}(s)+\frac{1}{\Delta_{1}} \int_{0}^{1} h_{1}(s) d H(\tau)=h_{1}(s)\left(1+\frac{1}{\Delta_{1}} \int_{0}^{1} d H(\tau)\right)=J_{1}(s) .
\end{aligned}
$$

(b) For the second inequality, we obtain

$$
\begin{aligned}
G_{1}(t, s) & \geq k_{1}(t) h_{1}(s)+\frac{t^{\alpha-1}}{\Delta_{1}} \int_{0}^{1} k_{1}(\tau) h_{1}(s) d H(\tau) \\
& =\frac{1}{\tau_{1}}\left(\tau_{1} h_{1}(s)\right)\left(k_{1}(t)+\frac{t^{\alpha-1}}{\Delta_{1}} \int_{0}^{1} k_{1}(\tau) d H(\tau)\right)=\gamma_{1}(t) J_{1}(s) .
\end{aligned}
$$

We observe that $\gamma_{1}(t)>0$ for all $t \in(0,1)$, and if $H \not \equiv$ const., then $\gamma_{1}(1)>0$.

Lemma 2.7 Assume that $H:[0,1] \rightarrow \mathbb{R}$ is a nondecreasing function, $\Delta_{1}>0$ and $z \in$ $C([0,1]), z(t) \geq 0$ for all $t \in[0,1]$. Then the solution $u(t), t \in[0,1]$ of problem (1)-(2) satisfies the inequality $u(t) \geq \gamma_{1}(t) \max _{t^{\prime} \in[0,1]} u\left(t^{\prime}\right)$ for all $t \in[0,1]$.

Proof For $t \in[0,1]$, we obtain

$$
\begin{aligned}
u(t) & =\int_{0}^{1} G_{1}(t, s) z(s) d s \geq \int_{0}^{1} \gamma_{1}(t) J_{1}(s) z(s) d s=\gamma_{1}(t) \int_{0}^{1} J_{1}(s) z(s) d s \\
& \geq \gamma_{1}(t) \int_{0}^{1} G_{1}\left(t^{\prime}, s\right) z(s) d s=\gamma_{1}(t) u\left(t^{\prime}\right), \quad \forall t, t^{\prime} \in[0,1] .
\end{aligned}
$$


Therefore, we deduce that $u(t) \geq \gamma_{1}(t) \max _{t^{\prime} \in[0,1]} u\left(t^{\prime}\right)$ for all $t \in[0,1]$.

We can also formulate similar results as Lemmas 2.3-2.7 above for the fractional differential equation

$$
D_{0+}^{\beta} v(t)+\widetilde{z}(t)=0, \quad 0<t<1, m-1<\beta \leq m,
$$

with the integral boundary conditions

$$
v(0)=v^{\prime}(0)=\cdots=v^{(m-2)}(0)=0, \quad v(1)=\int_{0}^{1} v(s) d K(s),
$$

where $m \in \mathbb{N}, m \geq 3, K:[0,1] \rightarrow \mathbb{R}$ is a nondecreasing function and $\widetilde{z} \in C([0,1])$. We denote by $\Delta_{2}, g_{2}, G_{2}, h_{2}, k_{2}, \tau_{2}, J_{2}$, and $\gamma_{2}$ the corresponding constants and functions for problem (6)-(7) defined in a similar manner as $\Delta_{1}, g_{1}, G_{1}, h_{1}, k_{1}, \tau_{1}, J_{1}$, and $\gamma_{1}$, respectively.

In the proof of our main result we shall use the following nonlinear alternative of LeraySchauder type (see [12]).

Theorem 2.1 Let $X$ be a Banach space with $\Omega \subset X$ closed and convex. Assume $U$ is a relatively open subset of $\Omega$ with $0 \in U$, and let $S: \bar{U} \rightarrow \Omega$ be a completely continuous operator (continuous and compact). Then either

(1) S has a fixed point in $\bar{U}$, or

(2) there exist $u \in \partial U$ and $v \in(0,1)$ such that $u=v S u$.

\section{Main result}

In this section, we investigate the existence of positive solutions for our problem (S)-(BC). We present now the assumptions that we shall use in the sequel.

(H1) $H, K:[0,1] \rightarrow \mathbb{R}$ are nondecreasing functions, $\Delta_{1}=1-\int_{0}^{1} s^{\alpha-1} d H(s)>0$, $\Delta_{2}=1-\int_{0}^{1} s^{\beta-1} d K(s)>0$.

(H2) The functions $f, g \in C([0,1] \times[0, \infty) \times[0, \infty),(-\infty,+\infty))$ and there exist functions $p_{1}, p_{2} \in C([0,1],(0, \infty))$ such that $f(t, u, v) \geq-p_{1}(t)$ and $g(t, u, v) \geq-p_{2}(t)$ for any $t \in[0,1]$ and $u, v \in[0, \infty)$.

(H3) $f(t, 0,0)>0, g(t, 0,0)>0$ for all $t \in[0,1]$.

We consider the system of nonlinear fractional differential equations

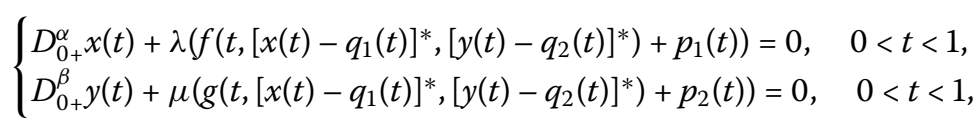

with the integral boundary conditions

$$
\begin{cases}x(0)=x^{\prime}(0)=\cdots=x^{(n-2)}(0)=0, & x(1)=\int_{0}^{1} x(s) d H(s) \\ y(0)=y^{\prime}(0)=\cdots=y^{(m-2)}(0)=0, & y(1)=\int_{0}^{1} y(s) d K(s)\end{cases}
$$

where

$$
z(t)^{*}= \begin{cases}z(t), & z(t) \geq 0 \\ 0, & z(t)<0\end{cases}
$$


and $\left(q_{1}, q_{2}\right)$ with $q_{1}(t)=\lambda \int_{0}^{1} G_{1}(t, s) p_{1}(s) d s, q_{2}(t)=\mu \int_{0}^{1} G_{2}(t, s) p_{2}(s) d s$ is the solution of the system of fractional differential equations

$$
\left\{\begin{array}{l}
D_{0+}^{\alpha} q_{1}(t)+\lambda p_{1}(t)=0, \quad 0<t<1, \\
D_{0+}^{\beta} q_{2}(t)+\mu p_{2}(t)=0, \quad 0<t<1,
\end{array}\right.
$$

with the integral boundary conditions

$$
\begin{cases}q_{1}(0)=q_{1}^{\prime}(0)=\cdots=q_{1}^{(n-2)}(0)=0, & q_{1}(1)=\int_{0}^{1} q_{1}(s) d H(s), \\ q_{2}(0)=q_{2}^{\prime}(0)=\cdots=q_{2}^{(m-2)}(0)=0, & q_{2}(1)=\int_{0}^{1} q_{2}(s) d K(s) .\end{cases}
$$

By (H2), we have $q_{1}(t)>0, q_{2}(t)>0$ for all $t \in(0,1)$.

We shall prove that there exists a solution $(x, y)$ for the boundary value problem (8)-(9) with $x(t) \geq q_{1}(t)$ and $y(t) \geq q_{2}(t)$ for all $t \in[0,1]$. In this case, the functions $u(t)=x(t)-q_{1}(t)$ and $v(t)=y(t)-q_{2}(t), t \in[0,1]$, represent a nonnegative solution, positive on $(0,1)$ of the boundary value problem (S)-(BC). Indeed, by (8)-(9) and (10)-(11), we have

$$
\begin{aligned}
D_{0+}^{\alpha} u(t)= & D_{0+}^{\alpha} x(t)-D_{0+}^{\alpha} q_{1}(t)=-\lambda f\left(t,\left[x(t)-q_{1}(t)\right]^{*},\left[y(t)-q_{2}(t)\right]^{*}\right) \\
& -\lambda p_{1}(t)+\lambda p_{1}(t)=-\lambda f(t, u(t), v(t)), \quad \forall t \in(0,1), \\
D_{0+}^{\beta} \nu(t)= & D_{0+}^{\beta} y(t)-D_{0+}^{\beta} q_{2}(t)=-\mu g\left(t,\left[x(t)-q_{1}(t)\right]^{*},\left[y(t)-q_{2}(t)\right]^{*}\right) \\
& -\mu p_{2}(t)+\mu p_{2}(t)=-\mu g(t, u(t), v(t)), \quad \forall t \in(0,1),
\end{aligned}
$$

and

$$
\begin{aligned}
& u(0)=x(0)-q_{1}(0)=0, \quad \ldots, \quad u^{(n-2)}(0)=x^{(n-2)}(0)-q_{1}^{(n-2)}(0)=0, \\
& v(0)=y(0)-q_{2}(0)=0, \quad \ldots, \quad v^{(m-2)}(0)=y^{(m-2)}(0)-q_{2}^{(m-2)}(0)=0, \\
& u(1)=x(1)-q_{1}(1)=\int_{0}^{1} x(s) d H(s)-\int_{0}^{1} q_{1}(s) d H(s)=\int_{0}^{1} u(s) d H(s), \\
& v(1)=y(1)-q_{2}(1)=\int_{0}^{1} y(s) d K(s)-\int_{0}^{1} q_{2}(s) d K(s)=\int_{0}^{1} v(s) d K(s) .
\end{aligned}
$$

Therefore, in what follows, we shall investigate the boundary value problem (8)-(9).

By using Lemma 2.3, the system (8)-(9) is equivalent to the system

$$
\begin{cases}x(t)=\lambda \int_{0}^{1} G_{1}(t, s)\left(f\left(s,\left[x(s)-q_{1}(s)\right]^{*},\left[y(s)-q_{2}(s)\right]^{*}\right)+p_{1}(s)\right) d s, & t \in[0,1] \\ y(t)=\mu \int_{0}^{1} G_{2}(t, s)\left(g\left(s,\left[x(s)-q_{1}(s)\right]^{*},\left[y(s)-q_{2}(s)\right]^{*}\right)+p_{2}(s)\right) d s, & t \in[0,1] .\end{cases}
$$

We consider the Banach space $X=C([0,1])$ with supremum norm $\|\cdot\|$ and the Banach space $Y=X \times X$ with the norm $\|(x, y)\|_{Y}=\|x\|+\|y\|$. We also define the cones

$$
\begin{aligned}
& P_{1}=\left\{x \in X, x(t) \geq \gamma_{1}(t)\|x\|, \forall t \in[0,1]\right\} \subset X, \\
& P_{2}=\left\{y \in X, y(t) \geq \gamma_{2}(t)\|y\|, \forall t \in[0,1]\right\} \subset X,
\end{aligned}
$$

and $P=P_{1} \times P_{2} \subset Y$. 
For $\lambda, \mu>0$, we define now the operator $\mathcal{Q}: P \rightarrow Y$ by $\mathcal{Q}(x, y)=\left(T_{1}(x, y), T_{2}(x, y)\right)$ with

$$
\begin{array}{ll}
T_{1}(x, y)(t)=\lambda \int_{0}^{1} G_{1}(t, s)\left(f\left(s,\left[x(s)-q_{1}(s)\right]^{*},\left[y(s)-q_{2}(s)\right]^{*}\right)+p_{1}(s)\right) d s, \quad 0 \leq t \leq 1, \\
T_{2}(x, y)(t)=\mu \int_{0}^{1} G_{2}(t, s)\left(g\left(s,\left[x(s)-q_{1}(s)\right]^{*},\left[y(s)-q_{2}(s)\right]^{*}\right)+p_{2}(s)\right) d s, \quad 0 \leq t \leq 1 .
\end{array}
$$

Lemma 3.1 If $(\mathrm{H} 1)$ and $(\mathrm{H} 2)$ hold, then the operator $\mathcal{Q}: P \rightarrow P$ is a completely continuous operator.

Proof The operators $T_{1}, T_{2}$ are well defined. For every $(x, y) \in P$, we have

$$
\begin{aligned}
& T_{1}(x, y)(t) \leq \lambda \int_{0}^{1} J_{1}(s)\left(f\left(s,\left[x(s)-q_{1}(s)\right]^{*},\left[y(s)-q_{2}(s)\right]^{*}\right)+p_{1}(s)\right) d s<\infty, \\
& T_{2}(x, y)(t) \leq \mu \int_{0}^{1} J_{2}(s)\left(g\left(s,\left[x(s)-q_{1}(s)\right]^{*},\left[y(s)-q_{2}(s)\right]^{*}\right)+p_{2}(s)\right) d s<\infty,
\end{aligned}
$$

for all $t \in[0,1]$, and

$$
\begin{aligned}
T_{1}(x, y)(t) & \geq \lambda \int_{0}^{1} \gamma_{1}(t) J_{1}(s)\left(f\left(s,\left[x(s)-q_{1}(s)\right]^{*},\left[y(s)-q_{2}(s)\right]^{*}\right)+p_{1}(s)\right) d s \\
& \geq \lambda \gamma_{1}(t) \int_{0}^{1} G_{1}\left(t^{\prime}, s\right)\left(f\left(s,\left[x(s)-q_{1}(s)\right]^{*},\left[y(s)-q_{2}(s)\right]^{*}\right)+p_{1}(s)\right) d s \\
& =\gamma_{1}(t) T_{1}(x, y)\left(t^{\prime}\right), \\
T_{2}(x, y)(t) & \geq \mu \int_{0}^{1} \gamma_{2}(t) J_{2}(s)\left(g\left(s,\left[x(s)-q_{1}(s)\right]^{*},\left[y(s)-q_{2}(s)\right]^{*}\right)+p_{2}(s)\right) d s \\
& \geq \mu \gamma_{2}(t) \int_{0}^{1} G_{2}\left(t^{\prime}, s\right)\left(g\left(s,\left[x(s)-q_{1}(s)\right]^{*},\left[y(s)-q_{2}(s)\right]^{*}\right)+p_{2}(s)\right) d s \\
& =\gamma_{2}(t) T_{2}(x, y)\left(t^{\prime}\right),
\end{aligned}
$$

for all $t, t^{\prime} \in[0,1]$. Therefore, we obtain

$$
T_{1}(x, y)(t) \geq \gamma_{1}(t)\left\|T_{1}(x, y)\right\|, \quad T_{2}(x, y)(t) \geq \gamma_{2}(t)\left\|T_{2}(x, y)\right\|, \quad \forall t \in[0,1]
$$

and $\mathcal{Q}(x, y)=\left(T_{1}(x, y), T_{2}(x, y)\right) \in P$.

By using standard arguments, we deduce that the operator $\mathcal{Q}: P \rightarrow P$ is a completely continuous operator (a compact operator, that is, it maps bounded sets into relatively compact sets, and it is continuous).

It is clear that $(x, y) \in P$ is a solution of problem (8)-(9) if and only if $(x, y)$ is a fixed point of $\mathcal{Q}$.

Theorem 3.1 Assume that (H1)-(H3) hold. Then there exist constants $\lambda_{0}>0$ and $\mu_{0}>0$ such that for any $\lambda \in\left(0, \lambda_{0}\right]$ and $\mu \in\left(0, \mu_{0}\right]$, the boundary value problem (S)-(BC) has at least one positive solution. 
Proof Let $\delta \in(0,1)$ be fixed. From (H3), there exists $R_{0}>0$ such that

$$
\begin{aligned}
& f(t, u, v) \geq \delta f(t, 0,0)>0, \quad g(t, u, v) \geq \delta g(t, 0,0)>0, \\
& \forall t \in[0,1], u, v \in\left[0, R_{0}\right] .
\end{aligned}
$$

We define

$$
\begin{aligned}
& \bar{f}\left(R_{0}\right)=\max _{0 \leq t \leq 1,0 \leq u, v \leq R_{0}}\left\{f(t, u, v)+p_{1}(t)\right\} \geq \max _{0 \leq t \leq 1}\left\{\delta f(t, 0,0)+p_{1}(t)\right\}>0, \\
& \bar{g}\left(R_{0}\right)=\max _{0 \leq t \leq 1,0 \leq u, v \leq R_{0}}\left\{g(t, u, v)+p_{2}(t)\right\} \geq \max _{0 \leq t \leq 1}\left\{\delta g(t, 0,0)+p_{2}(t)\right\}>0, \\
& c_{1}=\int_{0}^{1} J_{1}(s) d s>0, \quad c_{2}=\int_{0}^{1} J_{2}(s) d s>0, \\
& \lambda_{0}=\frac{R_{0}}{4 c_{1} \bar{f}\left(R_{0}\right)}>0, \quad \mu_{0}=\frac{R_{0}}{4 c_{2} \bar{g}\left(R_{0}\right)}>0 .
\end{aligned}
$$

We will show that for any $\lambda \in\left(0, \lambda_{0}\right]$ and $\mu \in\left(0, \mu_{0}\right]$, problem (8)-(9) has at least one positive solution.

So, let $\lambda \in\left(0, \lambda_{0}\right]$ and $\mu \in\left(0, \mu_{0}\right]$ be arbitrary, but fixed for the moment. We define the set $U=\left\{(x, y) \in P,\|(x, y)\|_{Y}<R_{0}\right\}$. We suppose that there exist $(x, y) \in \partial U\left(\|(x, y)\|_{Y}=R_{0}\right.$ or $\left.\|x\|+\|y\|=R_{0}\right)$ and $v \in(0,1)$ such that $(x, y)=v \mathcal{Q}(x, y)$ or $x=v T_{1}(x, y), y=v T_{2}(x, y)$.

Because

$$
\begin{aligned}
& {\left[x(t)-q_{1}(t)\right]^{*}=x(t)-q_{1}(t) \leq x(t) \leq R_{0}, \quad \text { if } x(t)-q_{1}(t) \geq 0,} \\
& {\left[x(t)-q_{1}(t)\right]^{*}=0, \quad \text { for } x(t)-q_{1}(t)<0, \forall t \in[0,1],} \\
& {\left[y(t)-q_{2}(t)\right]^{*}=y(t)-q_{2}(t) \leq y(t) \leq R_{0}, \quad \text { if } y(t)-q_{2}(t) \geq 0,} \\
& {\left[y(t)-q_{2}(t)\right]^{*}=0, \quad \text { for } y(t)-q_{2}(t)<0, \forall t \in[0,1],}
\end{aligned}
$$

then, for all $t \in[0,1]$, we obtain

$$
\begin{aligned}
x(t)= & \nu T_{1}(x, y)(t) \leq T_{1}(x, y)(t) \\
= & \lambda \int_{0}^{1} G_{1}(t, s)\left(f\left(s,\left[x(s)-q_{1}(s)\right]^{*},\left[y(s)-q_{2}(s)\right]^{*}\right)+p_{1}(s)\right) d s \\
\leq & \lambda \int_{0}^{1} G_{1}(t, s) \bar{f}\left(R_{0}\right) d s \leq \lambda \int_{0}^{1} J_{1}(s) \bar{f}\left(R_{0}\right) d s \leq \lambda_{0} c_{1} \bar{f}\left(R_{0}\right)=R_{0} / 4, \\
y(t)= & \nu T_{2}(x, y)(t) \leq T_{2}(x, y)(t) \\
& =\mu \int_{0}^{1} G_{2}(t, s)\left(g\left(s,\left[x(s)-q_{1}(s)\right]^{*},\left[y(s)-q_{2}(s)\right]^{*}\right)+p_{2}(s)\right) d s \\
& \leq \mu \int_{0}^{1} G_{2}(t, s) \bar{g}\left(R_{0}\right) d s \leq \mu \int_{0}^{1} J_{2}(s) \bar{g}\left(R_{0}\right) d s \leq \mu_{0} c_{2} \bar{g}\left(R_{0}\right)=R_{0} / 4 .
\end{aligned}
$$

Hence $\|x\| \leq R_{0} / 4$ and $\|y\| \leq R_{0} / 4$. Then $R_{0}=\|(x, y)\|_{1}=\|x\|+\|y\| \leq \frac{R_{0}}{4}+\frac{R_{0}}{4}=\frac{R_{0}}{2}$, which is a contradiction. 
Therefore, by Theorem 2.1 (with $\Omega=P$ ), we deduce that $\mathcal{Q}$ has a fixed point $(x, y) \in \bar{U} \cap P$. That is, $(x, y)=\mathcal{Q}(x, y) \Leftrightarrow x=T_{1}(x, y), y=T_{2}(x, y)$ and $\|x\|+\|y\| \leq R_{0}$, with $x(t) \geq \gamma_{1}(t)\|x\| \geq$ 0 and $y(t) \geq \gamma_{2}(t)\|y\| \geq 0$ for all $t \in[0,1]$.

Moreover, by (12), we obtain

$$
\begin{aligned}
x(t) & =T_{1}(x, y)(t)=\lambda \int_{0}^{1} G_{1}(t, s)\left(f\left(s,\left[x(s)-q_{1}(s)\right]^{*},\left[y(s)-q_{2}(s)\right]^{*}\right)+p_{1}(s)\right) d s \\
& \geq \lambda \int_{0}^{1} G_{1}(t, s)\left(\delta f(s, 0,0)+p_{1}(s)\right) d s>\lambda \int_{0}^{1} G_{1}(t, s) p_{1}(s) d s=q_{1}(t), \quad \forall t \in(0,1), \\
y(t) & =T_{2}(x, y)(t)=\mu \int_{0}^{1} G_{2}(t, s)\left(g\left(s,\left[x(s)-q_{1}(s)\right]^{*},\left[y(s)-q_{2}(s)\right]^{*}\right)+p_{2}(s)\right) d s \\
& \geq \mu \int_{0}^{1} G_{2}(t, s)\left(\delta g(s, 0,0)+p_{2}(s)\right) d s>\mu \int_{0}^{1} G_{2}(t, s) p_{2}(s) d s=q_{2}(t), \quad \forall t \in(0,1) .
\end{aligned}
$$

Therefore, $x(t)>q_{1}(t)>0$ and $y(t)>q_{2}(t)>0$ for all $t \in(0,1)$.

Let $u(t)=x(t)-q_{1}(t) \geq 0$ and $v(t)=y(t)-q_{2}(t) \geq 0$ for all $t \in[0,1]$, with $u(t)>0, v(t)>0$ on $(0,1)$. Then $(u, v)$ is a positive solution of the boundary value problem (S)-(BC).

\section{Examples}

Let $\alpha=\frac{5}{2}(n=3), \beta=\frac{10}{3}(m=4)$,

$$
H(t)= \begin{cases}0, & t \in[0,1 / 4), \\ 3, & t \in[1 / 4,3 / 4), \\ 7 / 2, & t \in[3 / 4,1]\end{cases}
$$

and $K(t)=t^{4}$ for all $t \in[0,1]$. Then $\int_{0}^{1} u(s) d H(s)=3 u\left(\frac{1}{4}\right)+\frac{1}{2} u\left(\frac{3}{4}\right)$ and $\int_{0}^{1} v(s) d K(s)=$ $4 \int_{0}^{1} s^{3} v(s) d s$.

We consider the system of fractional differential equations

$$
\left\{\begin{array}{l}
D_{0+}^{5 / 2} u(t)+\lambda f(t, u(t), v(t))=0, \quad 0<t<1 \\
D_{0+}^{10 / 3} v(t)+\mu g(t, u(t), v(t))=0, \quad 0<t<1
\end{array}\right.
$$

with the boundary conditions

$$
\left\{\begin{array}{l}
u(0)=u^{\prime}(0)=0, \quad u(1)=3 u\left(\frac{1}{4}\right)+\frac{1}{2} u\left(\frac{3}{4}\right), \\
v(0)=v^{\prime}(0)=v^{\prime \prime}(0)=0, \quad v(1)=4 \int_{0}^{1} s^{3} v(s) d s .
\end{array}\right.
$$

Then we obtain $\Delta_{1}=1-\int_{0}^{1} s^{3 / 2} d H(s)=1-3\left(\frac{1}{4}\right)^{3 / 2}-\frac{1}{2}\left(\frac{3}{4}\right)^{3 / 2}=\frac{10-3 \sqrt{3}}{16} \approx 0.3002>0, \Delta_{2}=$ $1-\int_{0}^{1} s^{7 / 3} d K(s)=1-4 \int_{0}^{1} s^{16 / 3} d s=\frac{7}{19} \approx 0.3684>0$.

We also deduce

$$
\begin{aligned}
& g_{1}(t, s)=\frac{4}{3 \sqrt{\pi}} \begin{cases}t^{3 / 2}(1-s)^{3 / 2}-(t-s)^{3 / 2}, & 0 \leq s \leq t \leq 1, \\
t^{3 / 2}(1-s)^{3 / 2}, & 0 \leq t \leq s \leq 1,\end{cases} \\
& g_{2}(t, s)=\frac{1}{\Gamma(10 / 3)} \begin{cases}t^{7 / 3}(1-s)^{7 / 3}-(t-s)^{7 / 3}, & 0 \leq s \leq t \leq 1, \\
t^{7 / 3}(1-s)^{7 / 3}, & 0 \leq t \leq s \leq 1,\end{cases}
\end{aligned}
$$


$\tau_{1}=\frac{633+168 \sqrt{3}}{73}, h_{1}(s)=\frac{2}{\sqrt{\pi}} s(1-s)^{3 / 2}, J_{1}(s)=\tau_{1} h_{1}(s)=\frac{1266+336 \sqrt{3}}{73 \sqrt{\pi}} s(1-s)^{3 / 2}, s \in[0,1], \tau_{2}=$ $\frac{26}{7}, h_{2}(s)=\frac{1}{\Gamma(7 / 3)} s(1-s)^{7 / 3}, J_{2}(s)=\tau_{2} h_{2}(s)=\frac{26}{7 \Gamma(7 / 3)} s(1-s)^{7 / 3}, s \in[0,1], c_{1}=\int_{0}^{1} J_{1}(s) d s \approx$ 1.63225815 , and $c_{2}=\int_{0}^{1} J_{2}(s) d s \approx 0.2159704$.

Example 1 We consider the functions

$$
\begin{aligned}
& f(t, u, v)=(u-a)(u-b)+\cos \left(\theta_{1} v\right), \quad g(t, u, v)=(v-c)(v-d)+\sin \left(\theta_{2} u\right), \\
& t \in[0,1], u, v \geq 0
\end{aligned}
$$

where $b>a>0, d>c>0, \theta_{1}, \theta_{2}>0$.

There exists $M_{0}>0$ such that $f(t, u, v)+M_{0} \geq 0, g(t, u, v)+M_{0} \geq 0\left(p_{1}(t)=p_{2}(t)=M_{0}\right.$, $\forall t \in[0,1])$ for all $t \in[0,1], u, v \geq 0$. Indeed, $M_{0}=\max \left\{\frac{(b-a)^{2}}{4}+1, \frac{(d-c)^{2}}{4}+1\right\}$ satisfies the above inequalities.

Let $\delta=\min \left\{\frac{a(2 b-a)}{4(a b+1)}, \frac{c(2 d-c)}{4 c d}\right\}<1$ and $R_{0}=\min \left\{\frac{a}{2}, \frac{c}{2}, \frac{\pi}{2 \theta_{1}}, \frac{\pi}{2 \theta_{2}}\right\}$. Then

$$
f(t, u, v) \geq \delta f(t, 0,0)=\delta(a b+1), \quad g(t, u, v) \geq \delta g(t, 0,0)=\delta c d,
$$

for all $t \in[0,1], u, v \in\left[0, R_{0}\right]$. Besides,

$$
\begin{aligned}
& \bar{f}\left(R_{0}\right)=\max _{0 \leq t \leq 1,0 \leq u, v \leq R_{0}}\left\{f(t, u, v)+p_{1}(t)\right\}=a b+1+M_{0}, \\
& \bar{g}\left(R_{0}\right)=\max _{0 \leq t \leq 1,0 \leq u, v \leq R_{0}}\left\{g(t, u, v)+p_{2}(t)\right\}=c d+\sin \left(\theta_{2} R_{0}\right)+M_{0} .
\end{aligned}
$$

Then $\lambda_{0}=\frac{R_{0}}{4 c_{1}\left(a b+1+M_{0}\right)}$ and $\mu_{0}=\frac{R_{0}}{4 c_{2}\left(c d+\sin \left(\theta_{2} R_{0}\right)+M_{0}\right)}$. For example, if $a=1, b=2, c=3$, $d=4, \theta_{1}=\theta_{2}=1$, then $R_{0}=1 / 2, \delta=1 / 4, M_{0}=5 / 4, \bar{f}\left(R_{0}\right)=4.25, \bar{g}\left(R_{0}\right) \approx 13.72942553$, $\lambda_{0}=\frac{1}{34 c_{1}} \approx 0.01801906$, and $\mu_{0}=\frac{1}{8(12+\sin (1 / 2)+5 / 4) c_{2}} \approx 0.04215638$.

By Theorem 3.1, for any $\lambda \in\left(0, \lambda_{0}\right]$ and $\mu \in\left(0, \mu_{0}\right]$, we deduce that problem $\left(\mathrm{S}_{0}\right)-\left(\mathrm{BC}_{0}\right)$ has a positive solution $(u, v)$, with $\|(u, v)\| \leq R_{0}$.

Example 2 We consider the functions

$$
f(t, u, v)=v^{a}+\cos \left(\theta_{1} u\right), \quad g(t, u, v)=u^{b}+\cos \left(\theta_{2} v\right), \quad t \in[0,1], u, v \geq 0,
$$

where $a, b, \theta_{1}, \theta_{2}>0$.

There exists $M_{0}>0\left(M_{0}=1\right)$ such that $f(t, u, v)+M_{0} \geq 0, g(t, u, v)+M_{0} \geq 0\left(p_{1}(t)=\right.$ $\left.p_{2}(t)=M_{0}, \forall t \in[0,1]\right)$ for all $t \in[0,1], u, v \geq 0$.

Let $\delta=\frac{1}{2}<1$ and $R_{0}=\min \left\{\frac{\pi}{3 \theta_{1}}, \frac{\pi}{3 \theta_{2}}\right\}$. Then

$$
f(t, u, v) \geq \delta f(t, 0,0)=\frac{1}{2}, \quad g(t, u, v) \geq \delta g(t, 0,0)=\frac{1}{2}, \quad \forall t \in[0,1], u, v \in\left[0, R_{0}\right]
$$

Besides,

$$
\begin{aligned}
& \bar{f}\left(R_{0}\right)=\max _{0 \leq t \leq 1,0 \leq u, v \leq R_{0}}\left\{f(t, u, v)+p_{1}(t)\right\}=R_{0}^{a}+2, \\
& \bar{g}\left(R_{0}\right)=\max _{0 \leq t \leq 1,0 \leq u, v \leq R_{0}}\left\{g(t, u, v)+p_{2}(t)\right\}=R_{0}^{b}+2 .
\end{aligned}
$$


Then $\lambda_{0}=\frac{R_{0}}{4 c_{1}\left(R_{0}^{a}+2\right)}$ and $\mu_{0}=\frac{R_{0}}{4 c_{2}\left(R_{0}^{b}+2\right)}$. For example, if $a=2, b=1 / 2, \theta_{1}=\theta_{2}=1$, then $R_{0}=\frac{\pi}{3}, \bar{f}\left(R_{0}\right)=\frac{\pi^{2}}{9}+2, \bar{g}\left(R_{0}\right)=\sqrt{\frac{\pi}{3}}+2, \lambda_{0}=\frac{3 \pi}{4\left(\pi^{2}+18\right) c_{1}} \approx 0.05179543$, and $\mu_{0}=\frac{\pi}{4(\sqrt{3 \pi}+6) c_{2}} \approx$ 0.40094916 .

By Theorem 3.1, for any $\lambda \in\left(0, \lambda_{0}\right]$ and $\mu \in\left(0, \mu_{0}\right]$, we deduce that problem $\left(\mathrm{S}_{0}\right)-\left(\mathrm{BC} \mathrm{C}_{0}\right)$ has a positive solution $(u, v)$, with $\|(u, v)\| \leq R_{0}$.

\section{Competing interests}

The authors declare that they have no competing interests.

Authors' contributions

Both authors contributed equally to this paper. Both authors read and approved the final manuscript.

\section{Author details}

${ }^{1}$ Department of Mathematics, Gh. Asachi Technical University, lasi, 700506, Romania. ${ }^{2}$ Faculty of Computer Engineering and Automatic Control, Gh. Asachi Technical University, lasi, 700050, Romania.

\section{Acknowledgements}

This work was supported by the CNCS grant PN-II-ID-PCE-2011-3-0557, Romania.

Received: 30 April 2014 Accepted: 13 June 2014 Published: 22 Jul 2014

\section{References}

1. Baleanu, D, Diethelm, K, Scalas, E, Trujillo, JJ: Fractional Calculus Models and Numerical Methods. Series on Complexity, Nonlinearity and Chaos. World Scientific, Boston (2012)

2. Das, S: Functional Fractional Calculus for System Identification and Controls. Springer, New York (2008)

3. Graef, JR, Kong, L, Kong, Q, Wang, M: Uniqueness of positive solutions of fractional boundary value problems with non-homogeneous integral boundary conditions. Fract. Calc. Appl. Anal. 15(3), 509-528 (2012)

4. Kilbas, AA, Srivastava, HM, Trujillo, JJ: Theory and Applications of Fractional Differential Equations. North-Holland Mathematics Studies, vol. 204. Elsevier, Amsterdam (2006)

5. Podlubny, I: Fractional Differential Equations. Academic Press, San Diego (1999)

6. Sabatier, J, Agrawal, OP, Machado, JAT (eds.): Advances in Fractional Calculus: Theoretical Developments and Applications in Physics and Engineering. Springer, Dordrecht (2007)

7. Samko, SG, Kilbas, AA, Marichev, Ol: Fractional Integrals and Derivatives. Theory and Applications. Gordon \& Breach, Yverdon (1993)

8. Henderson, J, Luca, R: Positive solutions for a system of nonlocal fractional boundary value problems. Fract. Calc. Appl. Anal. 16(4), 985-1008 (2013)

9. Henderson, J, Luca, R: Existence and multiplicity of positive solutions for a system of fractional boundary value problems. Bound. Value Probl. 2014, 60 (2014)

10. Yuan, C, Jiang, D, O'Regan, D, Agarwal, RP: Multiple positive solutions to systems of nonlinear semipositone fractiona differential equations with coupled boundary conditions. Electron. J. Qual. Theory Differ. Equ. 2012, 13 (2012)

11. Yuan, C: Multiple positive solutions for $(n-1,1)$-type semipositone conjugate boundary value problems of nonlinear fractional differential equations. Electron. J. Qual. Theory Differ. Equ. 2010, 36 (2010)

12. Agarwal, RP, Meehan, M, O'Regan, D: Fixed Point Theory and Applications. Cambridge University Press, Cambridge (2001)

10.1186/1687-1847-2014-179

Cite this article as: Luca and Tudorache: Positive solutions to a system of semipositone fractional boundary value problems. Advances in Difference Equations 2014, 2014:179

\section{Submit your manuscript to a SpringerOpen ${ }^{\ominus}$ journal and benefit from:}

- Convenient online submission

- Rigorous peer review

- Immediate publication on acceptance

Open access: articles freely available online

- High visibility within the field

- Retaining the copyright to your article 